\title{
The Study on the Optimism and the Amount of Trust in Social Media Content and the Level of their Ethical Health among the Students of Sirjan University of Technology
}

\author{
S. KHAJEHASANI \\ Department of computer science, Sirjan \\ university of technology, Sirjan, P.O, Box \\ 78137-33385, Iran \\ L. DEHYADEGARI* \\ Department of computer science, Sirjan \\ university of technology, Sirjan, P.O, Box \\ 78137-33385, Iran \\ khajeh@sirjantech.ac.ir \\ I.dehyadegari@sirjantech.ac.ir*
}

\begin{abstract}
As trust in the physical space is an interactive element, the interaction in the internet as a space parallel to physical space, requires a level of trust especially the interpersonal trust. Perhaps we can argue that trust in the internet is more important, due to the acceptance of higher risk in the interaction with anonymous individuals. The effective mechanisms are applied in the internet financial transactions including trust in the trade marks, trust in the reputation of company or institution name/title, and trust in the secure technology used; however, in non-commercial interactions, the trust still has much higher risk. It originates from the need to interact with those whose identity is unknown to us. Thus, in this research we examine the amount of trust in the content of these social networks. This is a survey study and the research population consists of 200 students of Sirjan University of Technology that is randomly chosen. According to the statistical results obtained, there is no significant relationship between the time spent on virtual social networks and their daily use with trust in the social networks. However, there is a consistent significant relationship between the type of membership in virtual social networks and students' trust in them.
\end{abstract}

Key Words: Virtual Social Networks, Trust, Students of Sirjan University of Technology

\section{Introduction}

The virtual social networks are a new generation of community-based internet systems based on the norms of western societies that are increasingly expanding all over the world. In Iran too, since the emergence of these networks, their audience has grown steadily. Social networks with interactive features create a modern space in the field of social activism (Bravo, 2017). Therefore, it seems essential to know more about the nature of these media. There are two approaches to represent the individuals' identities regarding 
cyberspace (Golbeck, 2005). Rheingold believes that people in virtual communities do the same as what they do in their real life. Rheingold's view seems simplistic. In other words, people look for their ideals in the virtual world that cannot achieve in their real life and try to convince others that this is their real life and they live that way. They try to make not only the others' awareness but also their own "self-concept" based on the content that is presented by the virtual networks (CHinomona, 2016). Therefore, it is necessary to examine a number of hypotheses related to trust in virtual networks and the purpose of this research is to answer these hypotheses (SanaPour, 2011). These hypotheses are as follow:

1. What does social trust mean and what is its role and impact on the society and what are the factors that create and change it?

2. There is a significant relationship between the amount of trust in virtual networks and the amount of virtual networks use. The amount of trust in virtual networks is measured by the factors namely regarding the content of virtual social media as real, the amount of trust in the accuracy and authenticity of their news and information, the degree of the validity and acceptability of the virtual social network messengers, the amount of trust and optimism about the content of virtual networks compared to the internal media, the amount of trust in the identity and information of the others in social networks and the level of security while using virtual social networks, and the amount of virtual networks use includes the components of daily use, duration of use, and the type of membership in virtual social networks (Kwan, 2011).

\section{Research Background}

The researches related to trust in cyberspace in Iran are essentially done by focusing on e-business and ecommerce, including Dehghani's study. This study addresses the trust in the field of e-commerce and its relationship with security indicators (Dehghani, Firouzabadi, 2008). In the study done by Villegas, an understanding of how to build the consumer's trust to buy or use e-services is taken into account and after prioritizing the affecting factors on trust in e-services, they are evaluated based on a conceptual model on two service websites. This research model is based on interconnections. The method of research to measure trust in blog sphere is to use the structure of the links between weblogs and analyze their context. Thus, a webometric tool is used in this study. Trust-based control over social networks is the title of a research that is focused on the concerns about the access to the personal information in the social networks. In this study trust in social networks is classified according to the actors' calculations in social networks interconnections. Johnson in a research have measured trust indicators by examining the actors' communicative behavior in social networks interconnections. They have expanded the algorithms to measure the behavioral trust efficiently in their study (Johnson, 2011). Salehi have explored the concept of trust in online social networks pointing out that social networks have provided four new strategies to share, interact and meet each other, but these attractive and pleasant options have raise some concerns related to privacy, security, and in a word trust (Salehi, 2008). 


\section{Review of the First Hypothesis}

What does trust mean?

Trust is one of the important aspects of human relations and underlies the cooperation and collaboration among members of the community. Trust facilitates participation in various economic, political, social and cultural fields and increases individuals' willingness to collaborate with different social groups (Adali, 2010).

\section{Theoretical Foundations of Trust}

We can study trust and its related theories in three micro, macro and mid-levels. We can set different theories at each level that has different perspective on trust. In this paper we try to examine Anthony Giddens', James Coleman's and Francis Fukuyama's view as three representatives of these three levels (Bell, 2010).

\section{James Coleman (Micro Level)}

To Coleman trust is a central concept. In his view people carry out social actions to meet their needs and it is possible through social exchanges, and social exchanges thereby have a key role in doing social actions. He believes that there are at least two components in each trust-based relationship: trustor and trustee. It is assumed that both parts are targeted and seek to meet their needs. The trustor must decide whether to enter into a deal or action or not (i.e. to accept the risk or not) and the trustee must choose between maintaining trust and breaking it. Therefore, a trust-based relationship is a mutual act and is based on the principle of maximizing benefit in risky condition.

\section{Anthony Giddens (Mid-Level)}

Giddens in a discussion about trust, describes it as assurance of individuals' trustworthiness or reliance on an individual's quality or feature or the veracity of a phrase or words and sees it as an appropriate starting point to define this concept. Giddens believes that trust is directly associated with the concept of structure and institution on the one hand and the modern society features on the other hand. In his view, the structure is described in terms of rules and replicable resources. Replicability (repetitiveness) and continuity are the elements that he uses in the definition of trust. In Giddens perspective trust refers to the expectations fulfilled about probable events.

\section{Francis Fukuyama (Macro Level)}

Fukuyama deals with an economic approach to trust and its theoretical basis and focus is on social capital as a certain set of informal norms and values shared among members of a group that cooperate and collaborate with each other. Fukuyama argues that these values and norms include features like honesty, 
commitment and mutual relationships in a way that the individuals that collaborate with each other and participate in these norms and values with these features, create trust. Fukuyama believes that this collaboration is intended to increase economic efficiency and thus he considers trust based on social capital and in the field of economics and demonstrates the importance of trust in the structure of industrialized countries by suggesting that the structural difference among capitalist countries is due to their cultural characteristics and features and social capital which make members of the community trust each other and cooperate by forming new groups and corporations (Panahi, 2010).

\section{Research Method}

As mentioned before, this is a statistical research and the method of research to collect data is also questionnaire. The study population consists of 200 students of Sirjan University of Technology that is randomly selected and seems appropriate regarding the total population of the university. We show the statistical tables representing the membership period in these virtual networks to examine the first hypothesis and then present the statistical tables of response to each dimension of trust in virtual networks and finally test the second hypothesis. To test the second hypothesis, we use Pearson correlation test regarding the distance between all variables. Second hypothesis states that there is a correlation between the degree of trust in virtual networks and the amount of virtual social networks use. Table 5 shows the results of Pearson correlation test for this hypothesis.

\section{Research Findings}

Table 1 shows that most of the sample members have more than three years of membership in virtual networks. This group consists of 104 sample members comprising $52.5 \%$ of the research statistical sample. Another group includes the individuals that passed their membership period in virtual networks from 1 to 3 years and makes up $35.35 \%$ of the statistical sample with 70 members and finally those who have a membership period of less than one year in virtual networks and only a few who are 5 people have certified that they do not use these networks. Table 1 represents that more than half of the students examined have used these networks more than three years, indicating that these networks spread among these students.

Table 1. Duration of membership in virtual social networks

\begin{tabular}{|l|c|c|c|}
\hline & Abundance & Abundance percentage & $\begin{array}{c}\text { The cumulative } \\
\text { percentage }\end{array}$ \\
\hline Less than 1 year & 19 & 9.59 & 9.59 \\
\hline Between 1 to 3 years & 70 & 35.35 & 44.94 \\
\hline Between 3 to 5 years & 57 & 28.78 & 73.73 \\
\hline More than 5 years virtual & 47 & 23.73 & 97.47 \\
\hline $\begin{array}{l}\text { Don't use } \\
\text { networks }\end{array}$ & 5 & 2.52 & 100 \\
\hline
\end{tabular}


Table 2 shows the sample members' type of membership in the virtual networks. According to the statistics, about $80 \%$ of sample members use Telegram and Instagram and a few use other virtual networks.

Table 2. Frequency distribution of membership in virtual social networks among the respondents

\begin{tabular}{|l|l|l|}
\hline Social networks & Abundance & $\begin{array}{l}\text { Frequency distribution of } \\
\text { membership }\end{array}$ \\
\hline Telegram & 129 & 49.23 \\
\hline Instagram & 79 & 30.15 \\
\hline Whats up & 38 & 14.50 \\
\hline Viber & 15 & 5.72 \\
\hline Other & 1 & 0.38 \\
\hline
\end{tabular}

Table 3 shows that only 12.31 percent of the students under study use these networks less than an hour and the majority of them use these networks more than one hour, indicating that the daily use among these students is relatively high.

Table 3. The distribution of respondents by the amount of time spent using the social networks

\begin{tabular}{|l|c|c|c|}
\hline & Abundance & Valid percentage & The cumulative percentage \\
\hline Less than 1 hour & 25 & 12.31 & 12.31 \\
\hline Between 1 to 3 hours & 59 & 29.06 & 41.37 \\
\hline Between 3 to 5 hours & 72 & 35.46 & 76.84 \\
\hline More than 5 hours & 47 & 23.15 & 100 \\
\hline
\end{tabular}

By examination, the second hypothesis is supported, indicating that there is a correlation between the degree of trust in virtual networks and the amount of virtual social networks use. Table 4 and figure 1 show the students' response to the trust of virtual networks. 
Table 4. Students' response to the trust of virtual networks

\begin{tabular}{|l|c|c|c|c|c|}
\hline & $\begin{array}{c}\text { Very } \\
\text { low }\end{array}$ & Low & $\begin{array}{c}\text { Mediu } \\
\mathrm{m}\end{array}$ & Much & Average \\
\hline Actual Perception of Virtual Social Media Content & 33 & 67 & 75 & 20 & 47.2 \\
\hline $\begin{array}{l}\text { The amount of trust in the accuracy of news and information of } \\
\text { virtual networks }\end{array}$ & 39 & 70 & 83 & 6 & 45.2 \\
\hline Validity and acceptability of virtual networks' messengers & 35 & 89 & 65 & 9 & 44.4 \\
\hline $\begin{array}{l}\text { The amount of trust and optimism relative to the content of } \\
\text { virtual networks compared to the domestic media }\end{array}$ & 51 & 78 & 48 & 19 & 42.7 \\
\hline $\begin{array}{l}\text { The amount of trust in the identity and information that others } \\
\text { have for social networks }\end{array}$ & 43 & 90 & 52 & 12 & 42.7 \\
\hline Security level when using virtual social networks & 46 & 70 & 59 & 21 & 44.7 \\
\hline
\end{tabular}

Students' Response to the Trust of Virtual Networks

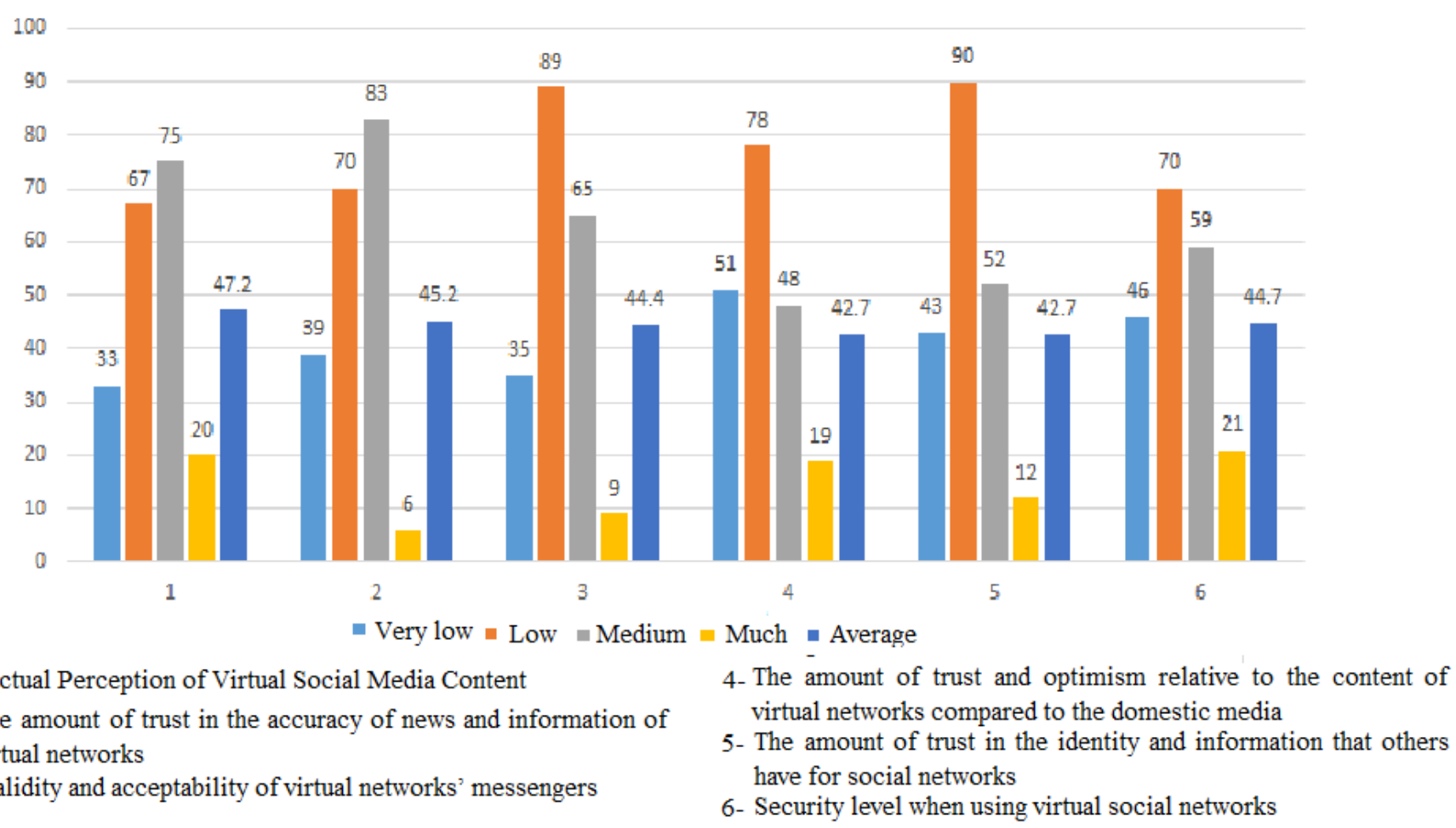

Figure 1. The Diagram of students' response to the trust of virtual networks 
Table 5 indicates Pearson correlation test results of variable of trust in social networks including sub-scales (the amount of regarding the content of virtual social media as real, the amount of trust in the accuracy and authenticity of their news and information, the degree of the validity and acceptability of virtual social network messengers, the amount of trust and optimism about the content of virtual networks compared to the internal media, the amount of trust in the identity and information of the others in social networks and the level of security while using virtual social networks), and dimensions of virtual social networks use (the amount of daily use, duration of networks use, and the type of membership in these networks). The significance level was set at 0.005. According to significance levels obtained, there is no significant relationship between the subscales of trust in social networks and duration of social networks use and the amount of these networks daily use. Therefore, considering that there is not a significant relationship between these variables, the intensity and direction of the relationship are not investigated. However, since the significant level between trust in virtual social networks and type of membership (Telegram, Instagram, WhatsApp, Viber) in virtual networks is 0.001 , there is a significant relationship between these two variables (Bell, 2010). As the relationship between trust in virtual networks and membership type in virtual networks is positive, and the obtained intensity is at medium level, we can conclude that individuals' trust in virtual social networks has a direct relationship with their trust in social networks. Figure 2 shows the diagram of Pearson correlation test results.

Table 5. Pearson correlation test results

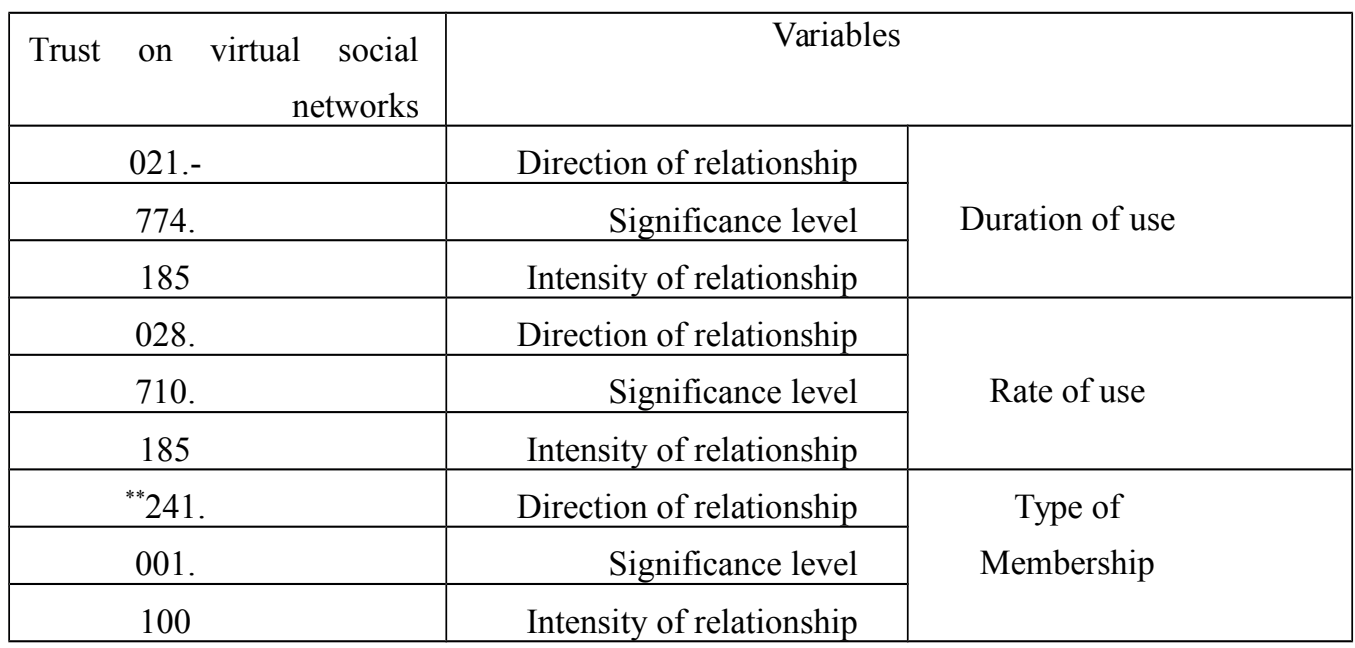




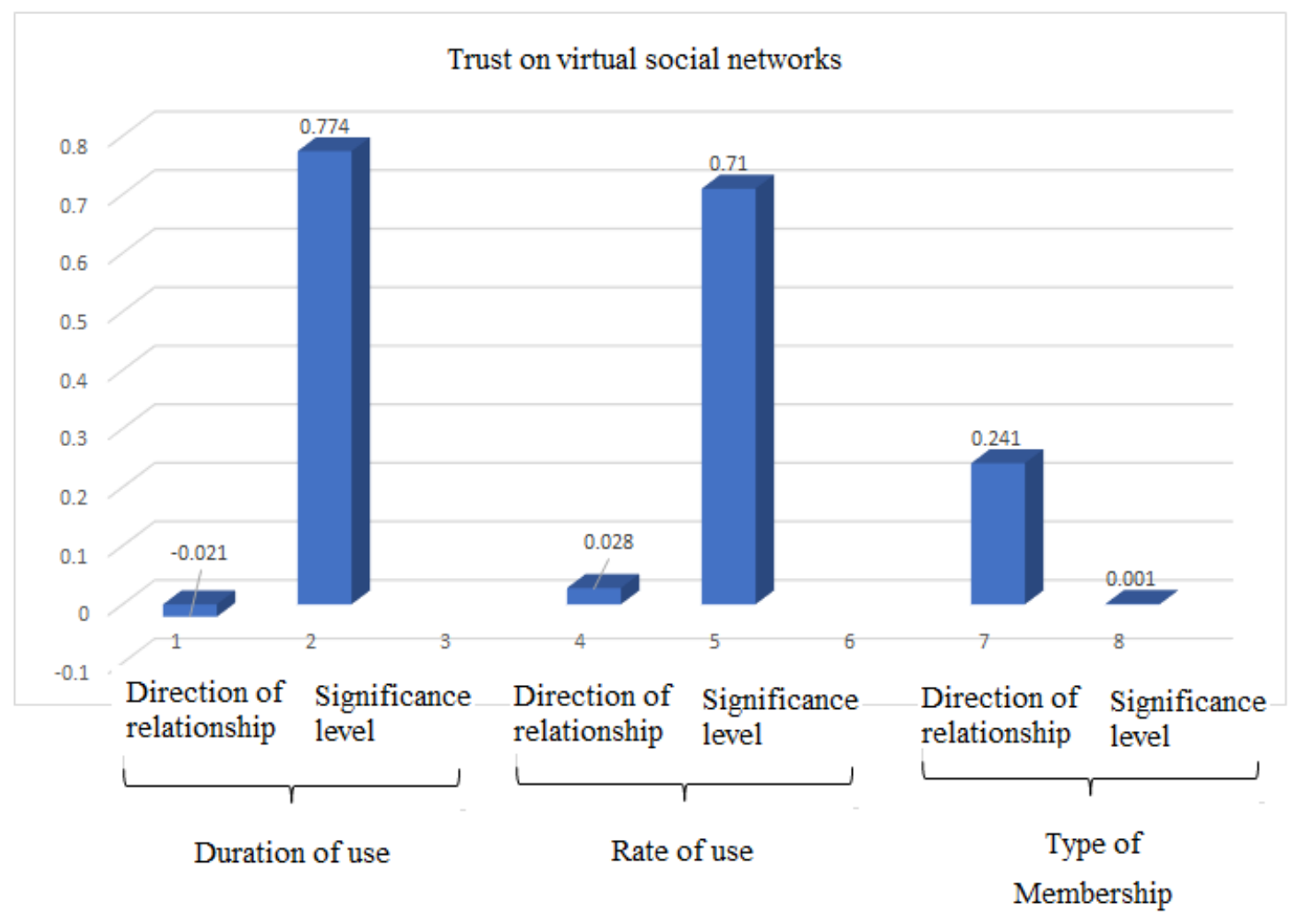

Figure 2. The diagram of Pearson correlation test results

\section{Conclusion}

It can be concluded from this research that virtual social networks have become significantly widespread among young people especially students. Regarding that the average of trust in virtual networks is 44.8 , we can conclude that the students have little trust in virtual networks, the validity of messages, their content, and the validity of virtual networks messengers. The results of research show that:

- The membership period in virtual social networks and the amount of their daily use have not been able to affect the amount of the students' trust in these networks.

- People's trust in virtual networks does not increase over time.

- Distrust cannot be the result of not being familiar with these networks.

- The results also show that the students' high daily use of these networks cannot be the reason for more trust in them and on the contrary low daily use of these networks do not lead to less trust in them. 
The only thing that affect the trust of students in virtual social networks is their membership type in these networks. The significant level between trust in virtual social networks and type of membership in virtual networks (0.001) shows that there is a significant relationship between these two variables. As the relationship between trust in virtual networks and membership type in virtual networks is positive, and the obtained intensity is at medium level, we can conclude that individuals' trust in virtual social networks has a direct relationship with their trust in social networks. Therefore, trust is affected by what type of virtual network is mostly used.

\section{Future works}

Continuing this research, the effects of social networks on other parameters of student life such as the relationship between the use of virtual social networks and students' social welfare or the impact of using social networks on student learning, can be evaluated. This research can also be extended to larger levels of society, such as other universities or high schools. The study of the impact of social network trust on ethical health in other ways with other cultures is also an interesting topic that can be considered in the future works.

\section{References}

1. SanaPour M., "Identity in the Age of Digital Media", Interdisciplinary Studies in Media, Research Institute for Humanities and Cultural Studies, Vol. 1, No. 2, pp. 130-139, 2011.

2. SalehiAmiri R., "Social Trust", Centre for Strategic Research of the Expediency Discernment Council, Cultural and Social Research, Vol. 8, No 16, 2008.

3. Bell D., "An Introduction to Cyber Cultures", Translated by Masoud Kosari and Hossein Hasani, Tehran: Sociologists Publication, 2010.

4. Panahi B., "Trust and Trust-Building in Organization", Peyke Noor, Vol 7, No 4, pp. 88-103, 2010.

5. Dehghani S., FirouzAbadi A., "Review of Security Measures in Cyber Space and its Impact on Increasing Customer's Trust", Thesis, MA, Shiraz University, Faculty of IT, 2008.

6. Johnson H., Lavesson N., Zhao H., Wu S., "On the Concept of Trust in Online Social Networks", In Trustworthy Internet, pp. 143-157, 2011.

7. Golbeck J., "Computing and Applying Trust in Web-based Social Networks", Unpublished doctoral dissertation, University of Maryland, College Park, 2005.

8. Golbeck J., "Trust and Nuanced Profile Similarity in Online Social Networks", ACM Transactions on the Web, Vol. 3, pp. 1-33, 2009.

9. Kwan M., Ramachandran D., "Trust and Online Reputation Systemse”, In J. Golbeck (Ed.), Computing with Social Trust, pp. 287-311, 2011.

10. Villegas W., "A trust-based access control scheme for social networks", Thesis: Master of Science, McGill University, School of Computer Science, 2008.

11. Adali S., Escriva R., Goldberg M. K., Hayvanovych M., Magdon Ismail M., Szymanski B. K., Wallace W. A., "Measuring Behavioral Trust in Social Networks", IEEE International Conference on (Trust), 2010.

12. Bravo R., Buil I., Chernatony L., Martínez E., "Managing brand identity: effects on the employees", International Journal of Bank Marketing, Vol.35, No. 1, pp. 1-34, 2017.

13. Chinomona R. "Brand communication, brand image and brand trust as antecedents of brand loyalty in Gauteng Province of South Africa", African Journal of Economic and Management Studies, Vol. 7, No. 1, pp. 1-28, 2016. 